\title{
CONTRACTURE OF THE VASTUS INTERMEDIUS IN CHILDREN
}

\author{
A Report of Two Cases \\ W. F. P. Gammie, J. Hermon Taylor and H. Urich, London, England \\ From the London Hospital
}

A condition affecting the quadriceps muscle in children causing limitation of flexion of the knee has been described by Hněvkovský (1961) and by Fairbank and Barrett (1961). Shortly after the appearance of these reports two children were seen in whom the clinical picture fitted the descriptions given by these authors and the diagnosis of progressive fibrosis of the vastus intermedius was confirmed by the subsequent findings. There have been no other reports of this condition.

Case 1-A girl, one of binovular twins, was born prematurely at the thirty-sixth week by spontaneous delivery. Apart from being slightly jaundiced on the fourth day she made normal progress until the age of five, when it was noticed that she could not bend her knees on walking upstairs, but had to waddle with a foot on either side of the stair carpet. The disability was an increasing handicap by the time she was brought to hospital at the age of ten years.

There was no history of injury and there was no family history of a similar condition. The maternal grandfather came from Eastern Germany. The twin sister, two older brothers and a younger sister were normal.

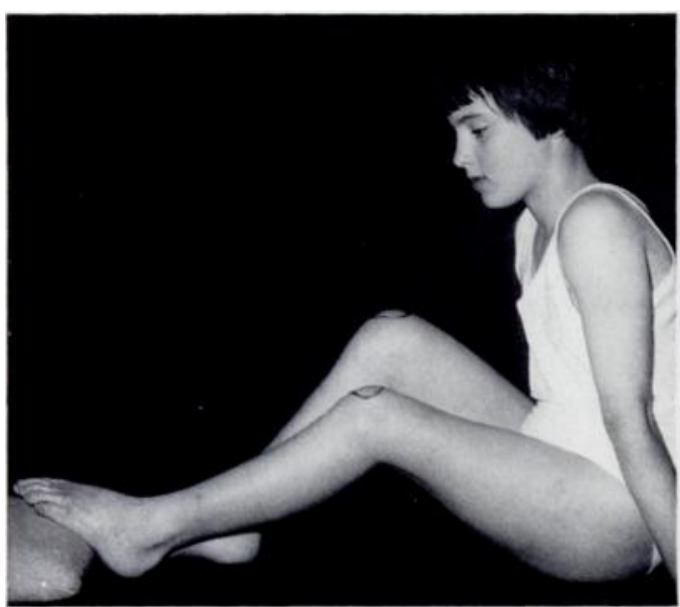

Fig. 1

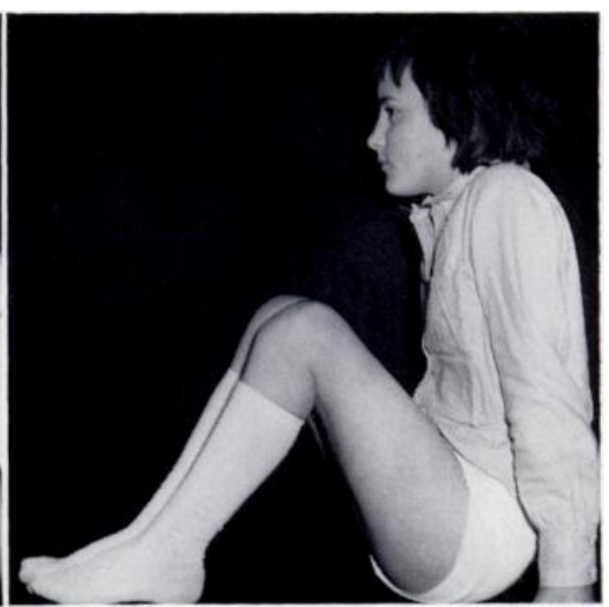

Fig. 2

Case 1. Figure 1-Knee flexion before operation. Figure 2-Knee flexion eight months after operation.

On examination the girl was alert and intelligent, entirely normal apart from her lower limbs. There was three-quarters of an inch of shortening of the left femur and one inch of wasting of the left thigh. The subcutaneous tissues of the thighs felt indurated, more so on the left than the right. Flexion was restricted, from 180 degrees to 140 degrees in the left knee and from 180 degrees to 90 degrees in the right (Fig. 1). A palpable, painless tenseness in the quadriceps prevented further forced flexion. The power of the free movement in both legs was normal and equal, and there was no evidence of any vascular or neurological abnormality. Both knees were normal clinically and radiologically but the left patella was a little smaller than the right. 
Investigations - The blood group was $\mathrm{O}$, Rhesus positive. The blood count, sedimentation rate, serum protein electrophoresis, serum creatine and creatinine and their excretion were all normal. Electromyography of the left quadriceps showed a reduced interference pattern in the vastus intermedius signifying fewer motor units with low amplitude action potentials reaching a peak of 300 to 500 microvolts. Normal patterns of large amplitude potentials were obtained from the vastus medialis, lateralis and rectus femoris.

At operation, in June 1961, an antero-medial incision was made on the left thigh and the rectus femoris was dissected off the vasti and retracted laterally. Flexion of the knee was demonstrated to be limited by the vastus intermedius, which was thickened, fibrotic and, at the limit of flexion, taut, but the vasti medialis and lateralis remained lax. Division of the vastus intermedius allowed the knee to flex to 85 degrees, but further flexion was limited by a tight band in the anterior part of the vastus lateralis. After this band had been divided flexion to 60 degrees was possible. After closure of the wound the knee was immobilised in plaster in 90 degrees of flexion.

The plaster was removed on the tenth day and active physiotherapy started; after a further week she was encouraged to walk. Subsequent progress was very satisfactory; two months later she was walking better and the active range of flexion in the left knee was from 180 degrees to 85 degrees. Eight months after operation she was able to run up and down stairs normally. At that time the scar was well healed, with neither tethering nor keloid formation. There was very slight weakness of extension of the left knee, in which there was a range of flexion from 180 degrees to 70 degrees. The range of the right knee was from 180 degrees to 75 degrees (Fig. 2).

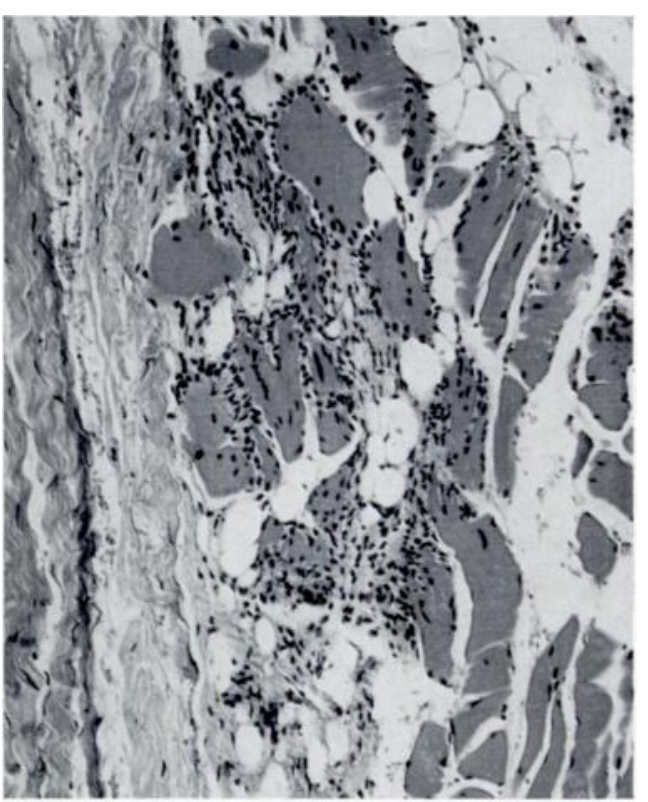

Fig. 3

Case 1-A section from the vastus intermedius showing a group of muscle fibres surrounded by young, highly cellular connective tissue. (Haematoxylin and eosin, $\therefore 115$.

Histology-Biopsies were made from each head of the quadriceps muscle. Histological examination of the vastus intermedius showed a considerable excess of collagen, partly in the form of dense fibrous strands which in places had a few buried muscle fibres, and partly in the form of loose fibro-fatty tissue in which the remaining muscle fibres were surrounded by highly cellular fibroblastic tissue with fine collagen fibrils (Fig. 3). The muscle fibres in these areas showed considerable distortion and some apparent fraying with indistinct transverse striation but no necrosis or phagocytosis. Elsewhere, the muscle was remarkably normal, showing only the slightest variation in the size of the muscle fibres, but a few of these were abnormally small. Longitudinal sections showed very slight crowding of sarcolemmal nuclei in places, but no proliferation of endomysial nuclei. No nerve fibres were seen. The other heads of the quadriceps muscle showed a similar slight variation in the size of muscle fibres but no excess of collagen and no replacement by adipose tissue.

Case 2-This girl, aged three, had been born by breech delivery at the thirty-sixth week after an antepartum haemorrhage from placenta praevia, and was the first of uniovular twins. She had passed a melaena stool ten hours after delivery and two days later had had a blood transfusion. Her subsequent development was normal and the same as that of her sister until she started walking at nineteen months. It was then noticed that she could not fully bend the left knee. This became more noticeable as she grew. Her sister remained normal. 
There was a history of twins on both sides of the family, but no history of a similar affliction. A brother had been born in January 1962 and was reported to be normal. There was no eastern European blood in the family.

Examination showed that the girl was healthy but walked with a stiff left knee and tended to swing the left hip outwards. There was no shortening of the leg or wasting of the thigh. The range of flexion was from 180 degrees to 120 degrees in the left knee and from 180 degrees to 60 degrees in the right knee (Fig. 4). Further flexion on the left was limited by tightness of the quadriceps muscle which was painless. Power within the range of movement was normal and the limb reflexes were brisk and equal. Radiographs of the knee were normal. The twin sister was found to have a normal range of movement in both knees. Their blood group was A, Rhesus positive. The results of other blood tests were identical in the two twins.

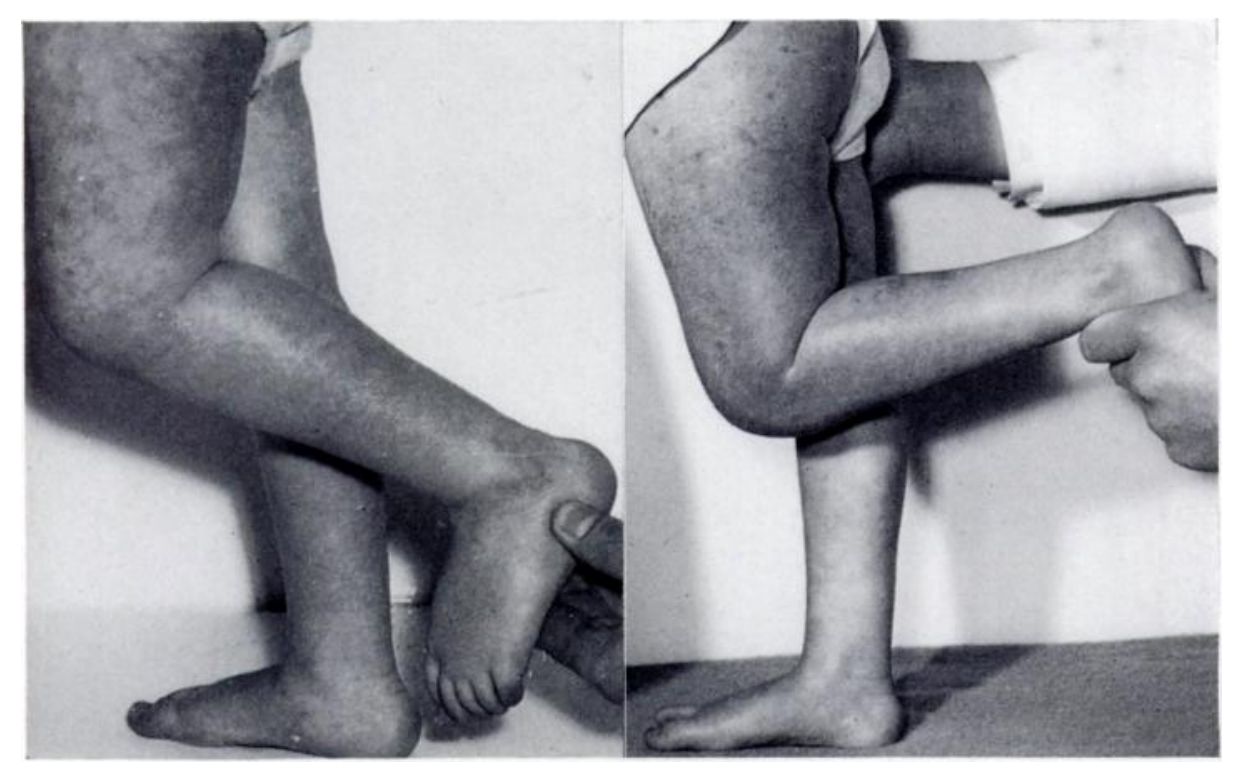

FIG. 4

FIG. 5

Case 2. Figure 4-Knee flexion before operation. Figure 5-Knee flexion three months after operation.

At operation, through an antero-medial incision, the rectus femoris was dissected free and the underlying vastus intermedius and lateralis found to be tight and fibrotic. These were divided together, as also was a tight band on the anterior border of the vastus medialis. Immediately the knee could be flexed to 85 degrees. After operation a plaster cylinder was applied with the knee at this angle.

The plaster was removed on the tenth day, when the child was able to flex the knee from 180 degrees to 90 degrees. She started to walk a week after the plaster had been removed.

Three months later her progress was good. She still dragged her left leg slightly. The scar was well healed and mobile. The range of flexion in the left knee had increased to 80 degrees (Fig. 5).

Histology - Biopsies were made of the vastusintermedius and the vastus lateralis. Histologically, considerable fibrosis was present in two samples from the vastus intermedius. In addition to thick fibrous strands, excess of collagen was present in numerous prominent fibrous septa separating the bundles of muscle fibres (Fig. 6). Bands of relatively acellular collagen were also found in the loose fibro-fatty tissue which replaced muscle in several areas. Part of the muscle consisted of abnormally thin fibres, with prominent but uniformly distributed sarcolemmal nuclei (Fig. 7). In some bundles these fibres showed approximately normal 
density, in others there was obvious loss of fibres with replacement by adipose tissue. Even in the most atrophic areas, where only a few isolated fibres ran through the fat, the transverse striations were well preserved. Nowhere did the nuclei show degenerative changes, such as pyknosis or clumping. Other parts of the muscle showed an apparently normal average

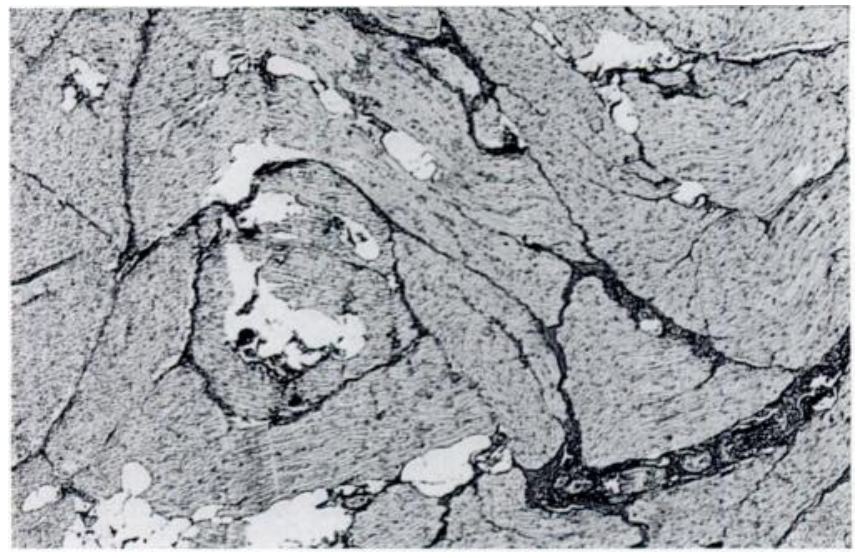

Fig. 6

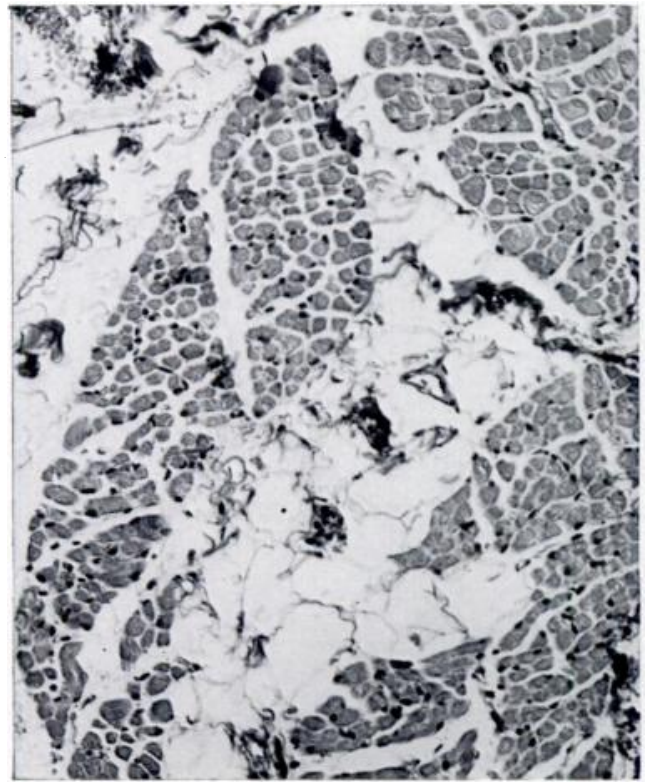

FIG. 7

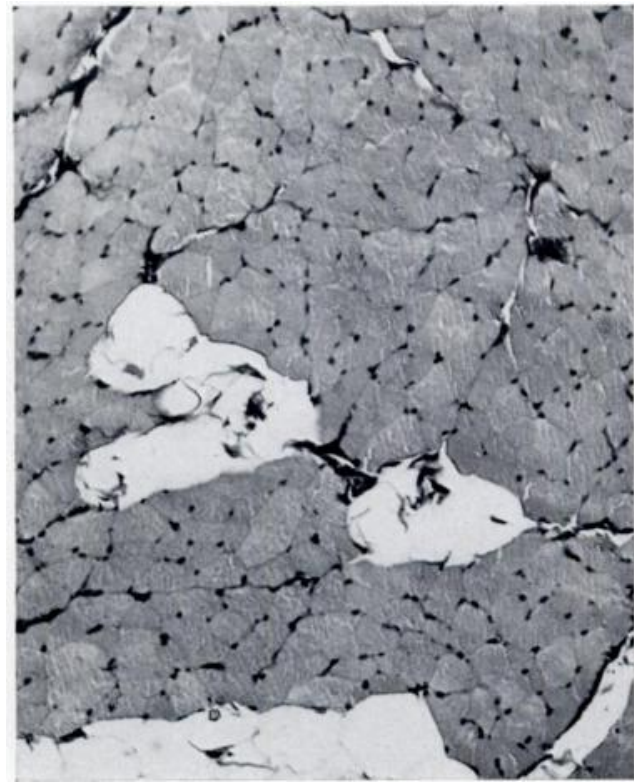

Fig. 8

Case 2. Figure 6-A section from the vastus intermedius showing prominent fibrous septa and infiltration of muscle bundles by fat. (Van Gieson, $\times 40$.) Figure 7-Another part of the same muscle, which consists of thin fibres. (Van Gieson, $\times 130$.) Figure 8-The same muscle showing normal fibres but with some replacement by fat. (Van Gieson, $\times 130$.)

diameter of fibres with some slight variation in size, but some of the bundles showed considerable infiltration by fatty tissue and interstitial fibrosis without obvious changes in individual muscle fibres (Fig. 8). Several small branches of peripheral nerves were seen in the preparations. No terminal or preterminal motor fibres could be found, but, on the other hand, one normal muscle spindle was seen as well as several sensory end-organs in the aponeurosis. A small biopsy of the vastus lateralis showed some variation in the size of muscle fibres and a slight excess of interstitial fat, but no obvious fibrosis. 


\section{DISCUSSION}

These patients were little different from those described by Hněvkovský (1961) and by Fairbank and Barrett (1961). The striking clinical features were the waddling gait, the progressive and painless inability to bend the knee, and the absence of pain on forced flexion. The condition may be unilateral or bilateral, and in the latter case the disability was asymmetrical.

The etiology of the condition is obscure. Histological examination of the affected muscle has thrown little light on the subject. In Hněvkovsky's cases there was narrowing and, in places, loss of muscle fibres, slight blurring of striation, replacement by fat, an increase in the number of endomysial nuclei and clusters of phagocytes were present. Fairbank and Barrett commented on the excess of fibrous and adipose tissue, the smallness of some muscle fibres, the abundance of endomysial nuclei and the good preservation of transverse striation. The remarkable striation of collagen observed by them remains a unique feature of their patient and the possibility of a fixation artefact suggested by the authors themselves cannot be ruled out.

Although the muscle fibres were affected differently in each of our two patients they shared with those previously described the common features of fibrosis and replacement of muscle by adipose tissue. In the older child, Case 1, the few surviving muscle fibres in the fibro-fatty tissue showed some fraying and loss of striation, and were surrounded by highly cellular and apparently young fibrous tissue, suggestive of activity and progression. In the younger child, Case 2 , there was nothing to suggest a progressive lesion but the uniformly small muscle fibres in one part, with excellent preservation of striation even in the most atrophic areas, was most striking; this was more uniform than is usually seen in denervation atrophy, and resembled more the foetal pattern found in myodysplasias. The significance of these discrepancies between the two patients is difficult to assess, and sampling errors, difference in age and the evolution of the lesion must all be taken into account.

Before the etiology of this condition can be established it must be known if the disease is progressive. Hněvkovský insisted that it was, and his histological findings as well as those in our first patient lend some support to this view. But there is little similarity to the commoner muscular dystrophies, although the possibility that the disease is a mild restricted myopathy with a tendency to fibrosis cannot be dismissed, and this would be strengthened if there was a genetic background. Fairbank and Barrett's patients were identical twins, but our younger patient was also one of identical twins and repeated examinations of the other twin failed to reveal any abnormality. Further observation is essential to exclude the possibility of delayed onset in the twin sister, because the occurrence in only one uniovular twin is strong evidence against a genetic factor. The disease is not peculiar to eastern Europe as suggested by Fairbank and Barrett. Our first patient had a German grandfather but the other was of pure British stock.

Hněvkovský also compared this condition with arthrogryposis, which is congenital but non-progressive. There is a histological similarity between some cases, such as our second patient, and the group of congenital contractures of muscles which includes, apart from amyoplasia congenita, such conditions as club foot, Sprengel's shoulder and congenital torticollis (Adams, Denny-Brown and Pearson 1953). They form a heterogeneous collection of anomalies, some of which may be associated with faulty development of innervation, while others appear to be primary abnormalities of muscle. Most are present at birth, with the exception of congenital torticollis, which is considered to be the result of birth injury, possibly through the mechanism of venous infarction.

Fairbank and Barrett thought that fibrosis of the vastus intermedius had more in common with contracture of the sternomastoid than with other diseases of this group, and the possibility of injury must be considered, and it is significant that at least four of the hitherto reported cases occurred in twins, both uniovular and binovular. It is difficult to reconcile birth injury with the etiology when the onset occurs at the age of five as in our first case, and at seven, 
as in one of Hněvkovský's patients. It is also difficult to understand why the condition should be progressive beyond the initial contraction of the scar tissue.

Whatever the nature of the disease, the disability itself is largely mechanical and can be relieved by surgery. This has varied from tendon lengthening by Hněvkovský, to excision of fibrosed muscle by Fairbank and Barrett and simple division of the muscle by us. The result of simple division is most satisfactory, but it is essential not only to divide the fibrous portion of the vastus intermedius but also any tight bands in the adjacent muscles. Immobilisation in plaster in flexion at a right angle or beyond for about ten days is sufficient, and the value of active physiotherapy thereafter cannot be overstressed. In our first case the range of active flexion in the first few weeks after operation was disappointing, but intensive physiotherapy regained the full range obtained at the operation.

The proper time for surgery is at the age of five or six. Hněvkovský reported an average age of onset of three years and an average age for surgery of six years. It is possible that our first patient would have made a more rapid and complete recovery if she had been operated on earlier, but as it was she was at an ideal age for co-operating with physiotherapy. On the other hand, our second patient might have done better if she had been operated on later when she would have been old enough to do her exercises.

It is probable that more patients with this condition will be seen. Serial electromyographic studies-difficult though they are in children-may throw some light on its eticlogy, as may histological studies of adequate biopsy material which should include branches of motor nerves. Thus we may learn more about this condition, which appears to be a distinct clinical entity lending itself well to surgery.

\section{SUMMARY}

1. Two patients are reported with progressive fibrosis of the vastus intermedius muscle causing limitation of flexion of the knee. The first patient, a girl of ten years who was one of binovular twins, noticed difficulty in walking at the age of five, the disability increasing with age. The other girl, aged three, was one of uniovular twins and displayed the abnormality as soon as she started walking.

2. The nature of the condition remains obscure.

3. Surgery is effective, and simple division of the affected muscle segment is recommended. A good range of passive flexion is obtained at once; the active range can be obtained thereafter by physiotherapy. Because this requires the patient's cooperation it is suggested that the best age for surgery is about five or six years.

Our thanks are due to $\mathrm{Mr} \mathrm{A}$. M. A. Moore and to $\mathrm{Mr} \mathrm{H}$. Osmond-Clarke for their permission to report the patients treated under their care, and to Professor 1 . Doniach for his helpful criticism. We are indebted to Mr R. F. Ruddick for the clinical photographs, and to Mr J. King for the photomicrographs.

\section{REFERENCES}

Adams, R. D., Denny-Brown, D., and Pearson, C. M. (1953): Diseases of Muscle. New York: Paul B. Hoeber Inc.

FaIRBANK, T. J., and BARretr, A. M. (1961): Vastus Intermedius Contracture in Early Childhood. Journal of Bone and Joint Surgery, 43-B, 326.

HNĚVkovskÝ, O. (1961): Progressive Fibrosis of the Vastus Intermedius Muscle in Children. Journal of Bone and Joint Surgery, 43-B, 318. 